\title{
Composición pigmentar de variantes amarillas y rojas de Ahnfeltia plicata (Ahnfeltiaceae, Rhodophyta) en la Región de Magallanes
}

\author{
Pigment composition of yellow and red variants of Ahnfeltia plicata \\ (Ahnfeltiaceae, Rhodophyta) in the Magellan Region
}

Fabio Méndez 1, 3, 4, Nair Yokoya ${ }^{2}$, Johanna Marambio ${ }^{1,3,4}$, Juan Pablo Rodriguez ${ }^{1,3,4}$ \& Andrés Mansilla1, 3,4

\section{Resumen}

La División Rhodophyta se caracteriza por tener representantes de macroalgas con valor comercial, debido a sus ficocoloides (carragenanos y agaranos). Ahnfeltia plicata, es una macroalga productora de agarosa y por lo tanto representa una oportunidad para la pesca artesanal y la industria. Praderas naturales de A. plicata observadas en Seno Skyring, Región de Magallanes, presentan variantes de color amarillas y rojas, que se adaptan ecofisiológicamente a las condiciones ambientales de sus hábitats. Uno de los parámetros fotosintéticos para evaluar el estado fisiológico de las macroalgas, es la concentración de pigmentos fotosintéticos. El objetivo del presente estudio, fue determinar las concentraciones de clorofila $\alpha$ y ficobiliproteínas, de variantes amarillas y rojas de A. plicata, que habitan en el sector de Seno Skyring, Región de Magallanes. Se realizó la extracción de pigmentos, mediante el uso de nitrógeno líquido y acetona al $90 \%$. Las variantes rojas presentaron las mayores concentraciones de pigmentos fotosintéticos en base a AFC $(287,443 \pm 202,045 \mathrm{ug} / \mathrm{g} \mathrm{MF} \pm$ $\mathrm{ES}) ; \mathrm{FC}(144,780 \pm 89,430 \mathrm{ug} / \mathrm{g} \mathrm{MF} \pm \mathrm{ES}) ; \mathrm{FE}$ $(367,850 \pm 219,185 \mathrm{ug} / \mathrm{g} \mathrm{MF} \pm \mathrm{ES})$ y clorofila $\alpha$ (Chl a: $61,975 \pm 219,185 \mathrm{ug} / \mathrm{g} \mathrm{MF} \pm \mathrm{ES}$ ). Estos resultados sugieren adaptabilidad a variaciones abióticas ambientales para la variante roja de $A$. plicata, derivado de una posible mayor capacidad de absorción de la energía luminosa.

\section{Palabras claves:}

Rhodophyta, Variantes cromáticas, Pigmentos, Región Subantártica.

\section{Abstract}

The Rhodophyta Division is characterized by having representatives of macroalgae with commercial value due to their phycocolloids. Ahnfeltia plicata It is a macroalgae producing agarose and therefore an opportunity for artisanal fisheries and industry. Natural meadows of $A$. plicata from Skyring Sound (Magellan Region), display yellow and red variants that should adapt eco-physiologically to environmental conditions of their habitats. The concentration of photosynthetic pigments is one of the parameters used to assess the physiological state of the seaweeds. The objective of this study was to determine the concentrations of chlorophyll $a$ and phycobiliproteins, in the yellow and red variants of $A$. plicata, inhabiting the Skyring Sound. The pigment extraction was performed using liquid nitrogen and acetone at $90 \%$. The

1 Laboratorio de Macroalgas Antárticas y Subantárticas (LMAS), Universidad de Magallanes, Punta Arenas, Chile.

2 Núcleo de Pesquisas em Ficologia, Instituto de Botânica Secretaria do Meio Ambiente de São Paulo, C. Postal 3005, 01031-970, São Paulo, SP, Brasil.

3 Instituto de Ecología y Biodiversidad, Santiago, Chile.

4 Universidad de Magallanes Departamento de Ciencias y Recursos Naturales, Laboratorio de Macroalgas Antarticas y Subantarticas, Punta Arenas, Chile. nyokoya@hotmail.com, johanna.marambio@yahoo.com, jprp_2@hotmail.cl, andres.mansilla@umag.cl, $>$ fabiomendezmansilla@gmail.com 
red variants have the highest concentrations of photosynthetic pigments such as AFC $(287,443$ $\pm 202,045 \mathrm{ug} / \mathrm{g}$ MF $\pm \mathrm{ES}) ; \mathrm{FC}(144,780 \pm$ $89,430 \mathrm{ug} / \mathrm{g} \mathrm{MF} \pm \mathrm{ES}) ; \mathrm{FE}(367,850 \pm 219,185$ $\mathrm{ug} / \mathrm{g} \mathrm{MF} \pm \mathrm{ES}$ ) and chlorophyll $\alpha$ (Chl $a: 61,975$ $\pm 219,185 \mathrm{ug} / \mathrm{g} \mathrm{MF} \pm \mathrm{ES})$. These results suggest adaptation of the red variant of $A$. plicata to the abiotic environmental conditions, thus allowing a possible greater absorptivity of light energy.

\section{Key words:}

Rhodophyta, color variants, pigments, Sub-Antarctic region.

\section{INTRODUCCIÓN}

Algunas especies de macroalgas pertenecientes a la División Rhodophyta presentan un importante valor comercial debido a la presencia de ficocoloides (agar y carragenanos), exclusivos de las macroalgas sin equivalentes sintéticos (Gómez, 2013), de amplia utilización en la industria química, agroalimentaria y productos biomédicos, utilizados como agentes gelificantes, espesantes y estabilizantes (Mansilla et al. 2012). Actualmente, la demanda de la industria nacional chilena por ficocoloides como materia prima sigue en aumento, siendo imperioso incorporar recursos alternativos para la extracción de ficocoloides, que ayuden a diversificar los recursos extraidos en la región, proporcionando nuevas fuentes de materia prima para la industria (Mansilla et al. 2012). Ahnfeltia plicata (Hudson) Fries 1836 (División Rhodophyta), es considerada una agarófita (Maggs \& Pueschel, 1989), que produce agarosa con importantes propiedades antioxidantes, antitumorales y anticoagulantes (Matsuhiro et al. 2014) generando una oportunidad para la pesca artesanal y la industria en la Región de Magallanes y Antártica Chilena y el país.

Ahnfeltia plicata, pertenece al orden Ahnfeltiales y a la familia Ahnfeltiaceae. Habita el intermareal bajo y el submareal somero, en sustratos rocosos dominados por bolones con alta exposición al oleaje (Ávila et al. 2012), crece hasta los 20 metros de profundidad formando extensas praderas marinas (Mansilla et al. 2013). Ahnfeltia plicata además cumple un importante rol ecológico, puesto que las extensas praderas albergan una gran cantidad de organismos como crustáceos, moluscos y anélidos (Ávila et al. 2012). Ahnfeltia plicata ha sido referida para ambos hemisferios, en regiones frías tales como Alaska, Noruega, Rusia y en Chile, principalmente en Valdivia, Puerto Natales, Seno Skyring, Seno Almirantazgo- Isla Grande de Tierra del Fuego, Región de Magallanes (Wiencke \& Clayton, 2002; Mansilla \& Navarro, 2003).

Particularmente se ha observado que en poblaciones naturales de A. plicata, localizadas en Seno Skyring (Región de Magallanes), se pueden distinguir dos fenotipos, individuos con coloración amarilla $e$ individuos con coloración roja (coloración normal de la especie). Estas variantes pigmentares, han sido observadas en otras especies de macroalgas, siendo utilizadas como marcadores para el aislamiento de cepas altamente productivas (Zhang \& Van der Meer, 1987; Niwa et al. 2002) y también para funciones fisiológicas, estudios bioquímicos de la composición del pigmento, historia de vida, procesos fotosintéticos, composición y crecimiento de polisacáridos (Kursar et al. 1983a, b; Ramus \& van der Meer, 1983; Costa \& Plastino, 2001; Guimarães et al. 2003; Yokoya et al. 2003). La aparición de tales cepas de colores se relaciona con la concentración relativa de pigmentos de clorofila a y pigmentos accesorios, como las ficobiliproteínas (aloficocianina, ficocianina y ficoeritrina) o a una deficiencia de estos últimos (Yokoya et al. 2007). La aparición de cepas de colores es común entre Rhodophytas, y han sido estudiadas en géneros como Gracilaria (C. Agardh) Greville, 1830 (Costa \& Plastino, 2011; Ursi \& Plastino, 2001); Eucheuma (N. L. Burman) Collins \& Hervey, 1917 y Kappaphycus (F.Schmitz) Doty ex P.C., 1996 (Gerung \& Ohno, 1997). Los individuos con diferentes composiciones de pigmentos pueden presentar variaciones en las respuestas bioquímicas y fisiológicas a factores abióticos (Kursar et al. 1983a, b; Yokoya et al. 2007), pueden presentar diferentes composiciones de polisacáridos (Guimarães, 2000), y diferentes tasas de crecimiento y patrones fotosintéticos (Guimarães, 2000; Ursi \& Plastino, 2001; Yokoya et al. 2007).

Los pigmentos fotosintéticos son compuestos 
particularmente diversos en las algas, los cuales son capaces de generar adaptaciones y aclimataciones que favorecen su sobrevivencia (Werlinger 2004, Plastino \& Mansilla, 2004). Por lo tanto, uno de los parámetros que permite evaluar el estado fisiológico de una especie de macroalga es la concentración de pigmentos fotosintéticos los cuales absorben eficientemente la luz emitida entre los 400-700 nm (Motten, 1995). Para la División Rhodophyta, se encuentran presente los pigmentos: clorofila a (440 y $675 \mathrm{~nm})$ y ficoeritrina (545 y $563 \mathrm{~nm}$ ), ficocianina (550 y $617 \mathrm{~nm})$ y aloficocianina (650 nm), (Werlinger, 2004; Gantt, 1990).

Debido a la importancia comercial de A. plicata, único genero del orden Ahnfeltiales de importancia economica (Mansilla et al. 2013), debido a la presencia del polisacárido agarosa, (Matsuhiro et al. 2014), es imperante la realización de investigación aplicada sobre su ecofisiología, además de estudios base sobre su crecimiento y producción de pigmentos orgánicos, entre otros, que permitan optimizar condiciones para su cultivo (salinidad, temperatura, irradiancia, etc.). El objetivo del presente estudio fue determinar las concentraciones de pigmentos de variantes amarillas y rojas de A. plicata, incluidas las ficobiliproteinas (aloficocianina, ficoeritrina $y$ ficocianina) y clorofila $a$, para la caracterización de ambas variantes de color que crecen en poblaciones naturales de Seno Skyring,
Ecorregión Subantártica de Magallanes.

\section{MATERIAL Y MÉTODOS}

\section{Área de estudio}

Ejemplares de coloración amarilla y roja (Fig. 1) fueron colectados en invierno 2012, los cuales crecen naturalmente en poblaciones de A. plicata en la localidad de Seno Skyring (52 39'21,7" S; 71³0'34,5" O), ubicada en Isla Riesco a 80 kilómetros de Punta Arenas, Región de Magallanes (Fig. 2). Las praderas de A. plicata en esta localidad están expuestas a vientos predominantes del noroeste a 2,5 metros aproximadamente de profundidad durante la pleamar.

\section{Procedimiento de muestreo y extracción de pigmentos}

Talos de A.plicata colectados en Seno Skyring fueron trasladados en un recipiente térmico al Laboratorio de Macroalgas Antárticas y Subantarticas (LMAS) de la Universidad de Magallanes (Pta. Arenas). Los talos fueron separados según su coloración (amarilla y roja). La extracción de pigmentos se realizó de acuerdo al protocolo de Kursar et al. (1983) con modificaciones de Plastino \& Guimarães, (2001). Se pesaron $300 \mathrm{mg}$. de seis talos de cada

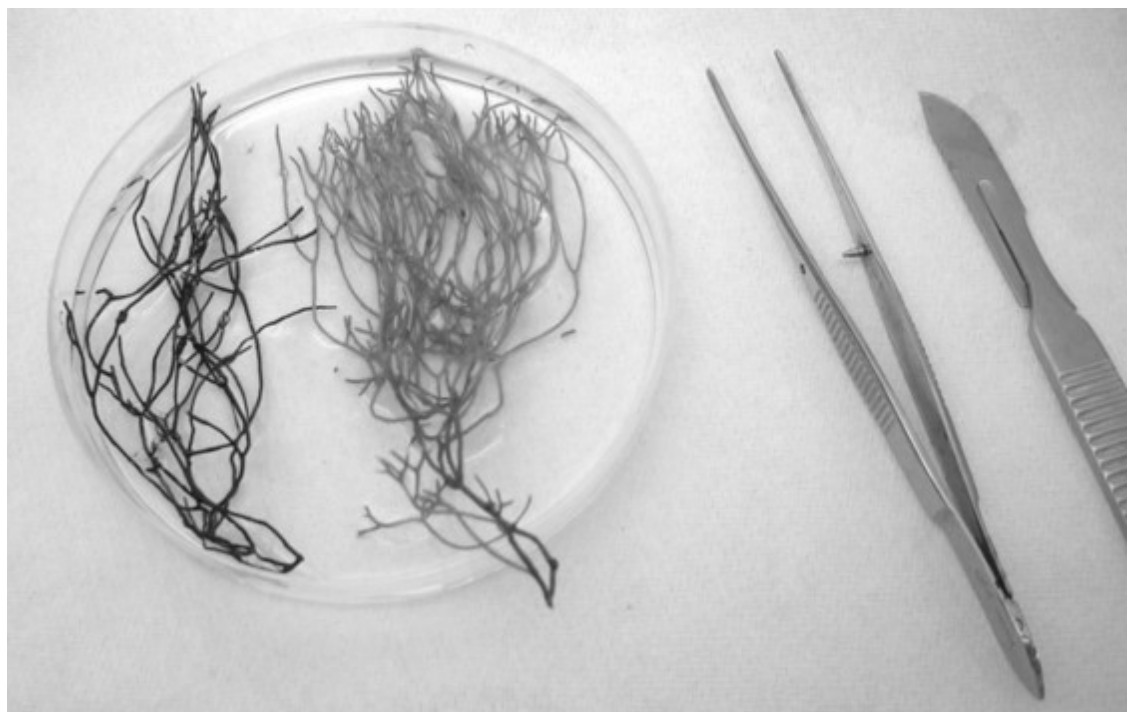

Fig. 1. Ejemplares de coloración amarilla y roja de Ahnfeltia plicata, colectadas del Sector de Seno Skyring, Región de Magallanes. 


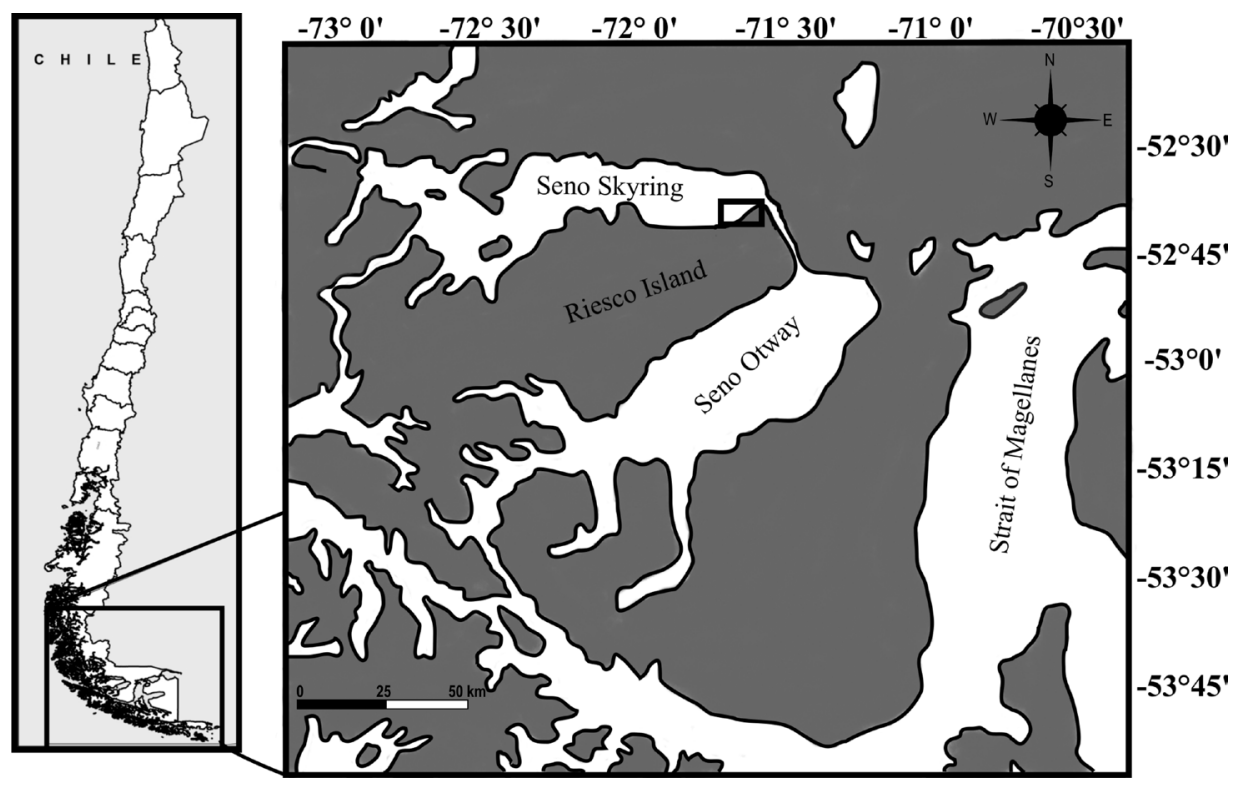

Fig. 2. Área de estudio de las praderas naturales de Ahnfeltia plicata en Seno Skyring, Región de Magallanes.

variante (amarilla y roja), los cuales fueron congelados $y$ triturados en un mortero con nitrógeno líquido. Posteriormente las muestras fueron depositadas en tubos de centrifuga a los que se le adicionó $1 \mathrm{~mL}$ de tampón fosfato (50 mM: pH 5,5 y T $\left.4^{\circ} \mathrm{C}\right)$. Luego se sometió a centrifugación a $11.000 \mathrm{rpm} ., 4^{\circ} \mathrm{C}$ y 20 min (Eppendorf Centrifuga 5804 R). Posterior al proceso de centrifugación, se extrajo el sobrenadante de los tubos de centrifuga para luego añadirlo en celdas de espectrofotometría, procediendo a la lectura de concentración de ficobiliproteinas (aloficocianina, ficoeritrina y ficocianina) en un espectrofotómetro (Espectrofotómetro SCINCO UV/Visible utilizándose para la medición de las absorbancias de pigmentos totales), con longitudes de onda de 498,5, 614 y $651 \mathrm{~nm}$., de acuerdo a la cuantificación de pigmentos descrita por Kursar et al. (1983). El precipitado resultante fue añadido en un tubo de centrifuga y se le adicionó acetona al 90\%. Luego se llevó nuevamente a centrifugación a 10.000 rpm, $4^{\circ} \mathrm{C}$ y 15 min. Posterior a este proceso se extrajo el sobrenadante depositándolo en su respectiva celda para la lectura de clorofila $\alpha$. Una vez en el espectrofotómetro se realizó la lectura con longitudes de onda de 630, 647 y 664 nm., de acuerdo a la cuantificación de pigmentos descrita por Jeffrey \& Humphrey, (1975).

Los máximos y datos de absorción de cada pigmento se obtuvieron con el programa Visionlite 5.0, para luego realizar la cuantificación de pigmentos de acuerdo a las siguientes formulaciones (Tabla 1).

Tabla 1. Fórmulas para la cuantificación de los pigmentos (AFC: aloficocianina, FC: ficocianina, FE: ficoeritrina, Chl a: clorofila a).

\begin{tabular}{|c|c|}
\hline \multicolumn{2}{|c|}{ Cuantificación de Pigmentos } \\
\hline Ficobiliproteínas & Ecuación \\
\hline Aloficocianina - AFC & $\mathrm{AFC}=181,3 \cdot \mathrm{A}_{651}-22,3 . \mathrm{A}_{614}$ \\
\hline Ficocianina - FC & $F C=151,1 . A_{614}-99,1 . A_{651}$ \\
\hline Ficoeritrina - FE & $F E=155,8 . A_{498,5}-40,0 . A_{614}-10,5 \cdot A_{651}$ \\
\hline (Kursar et al. 1983) & \\
\hline Clorofila a (Jeffrey \& Humphrey, 1975) & $\mathrm{Chl} \mathrm{a}=11.85 \mathrm{~A}_{664}-1.54 \mathrm{~A}_{647}-0.08 \mathrm{~A}_{630}$ \\
\hline
\end{tabular}


Los análisis de datos crudos y estadísticos se hicieron en Statistica ${ }^{\circledR}$ y Microsoft Office Excel 972003. La graficación de resultados del promedio ( $\pm \mathrm{DE}$, desviación estándar) de concentración pigmentar se realizaron en Microsoft Office Excel 97-2003. El análisis estadístico de los datos se realizó con un modelo no paramétrico de MannWhitney en el Software STATISTICA 7.1 V, con probabilidad $\mathrm{p}=0,05$.

\section{RESULTADOS}

Las concentraciones pigmentares para cada variante de A. plicata (amarillasy rojas) indicaron que la variante roja presenta una mayor concentración pigmentar en base a las AFC $(287,443 \pm 202,045$ $\mathrm{ug} / \mathrm{g} \mathrm{MF} \pm \mathrm{DE}), \mathrm{FC}(144,780 \pm 89,430 \mathrm{ug} / \mathrm{g}$ $\mathrm{MF} \pm \mathrm{DE}), \mathrm{FE}(367,850 \pm 219,185 \mathrm{ug} / \mathrm{g} \mathrm{MF} \pm$ DE) y Chl a $(61,975 \pm 219,185 \mathrm{ug} / \mathrm{g} \mathrm{MF} \pm \mathrm{DE})$, (Fig. 3). El pigmento fotosintético ficoeritrina fue el que presentó el mayor valor de concentración pigmentar (Fig. 3). El análisis estadístico, con el test del modelo no paramétrico de Mann-Whitney, indico que existen diferencias significativas en $\mathrm{Chl}$ a entre las variantes amarillas y rojas de A. plicata, con un valor de $\mathrm{p}=0,004$ (Tabla 2).

Respecto a las relaciones pigmentares para cada variante de A. plicata (amarillas y rojas), la variante amarilla presentó mayores valores para las relaciones FE/Chl $a(12,26), \mathrm{FC} / \mathrm{Chl} a(5,49)$ y AFC/Chl a $(15,05)$, mientras que la variante roja de A. plicata, presentó mayores valores para las relaciones de FE/FC (2,56), FE/AFC $(1,35), \mathrm{FC} /$ $\operatorname{AFC}(0,53)$ (Fig. 4).

Tabla 2. Análisis estadístico de los valores de concentración pigmentar de variantes amarillas y rojas de A. plicata. (AFC: aloficocianina, FC: ficocianina, FE: ficoeritrina, Chl a: clorofila a).

\begin{tabular}{cc}
\hline Variables & Valor $p$ \\
\hline Chl a & $0,004^{*}$ \\
FE & 0,078 \\
FC & 0,078 \\
AFC & 0,200 \\
\hline * $p>0,05$ &
\end{tabular}

\section{DISCUSIÓN Y CONCLUSIÓN}

Según Kirk (1994), las variaciones cromáticas pueden ser el resultado de una aclimatación fenotípica a distintas intensidades de luz (fotoaclimatación) o a variaciones genotípicas. Estas variaciones pueden observarse fácilmente en terreno, donde las praderas naturales de A. plicata en el sector de Seno Skyring, presentan variantes de coloración amarilla y roja. Generalmente en las estaciones donde la fertilidad es baja (otoñoinvierno), los talos poseen una coloración amarilla

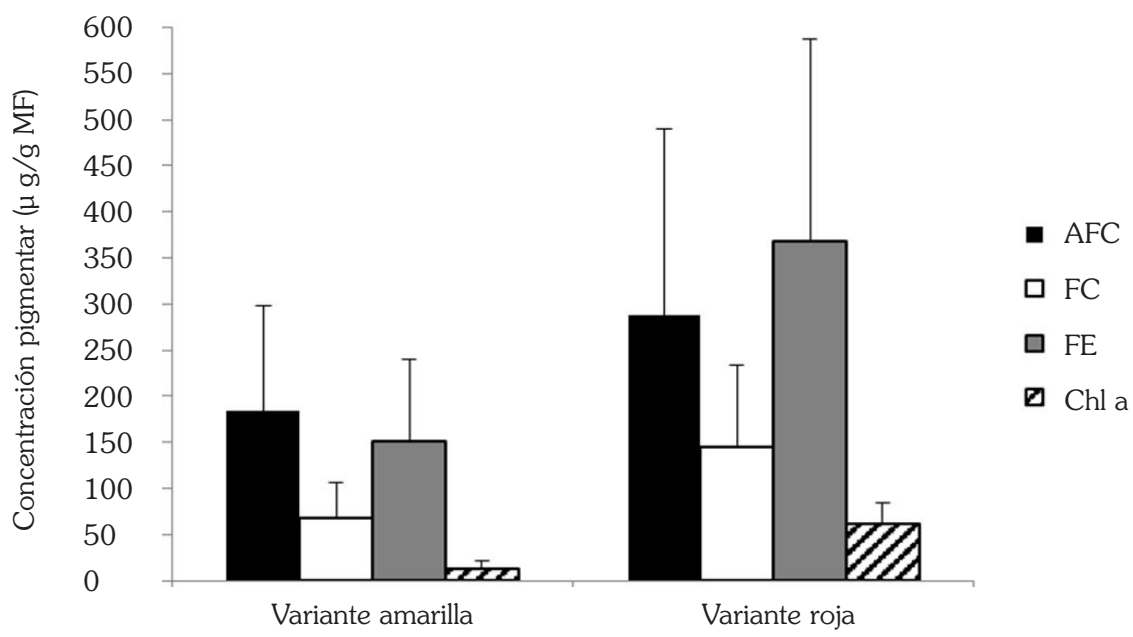

Fig. 3. Concentración pigmentar $(\mathrm{mg} / \mathrm{g})$ de variantes amarillas y rojas de A. plicata colectadas del Sector de Seno Skyring, Región de Magallanes. (AFC: aloficocianina, FC: ficocianina, FE: ficoeritrina, Chl $a$ : clorofila a). 


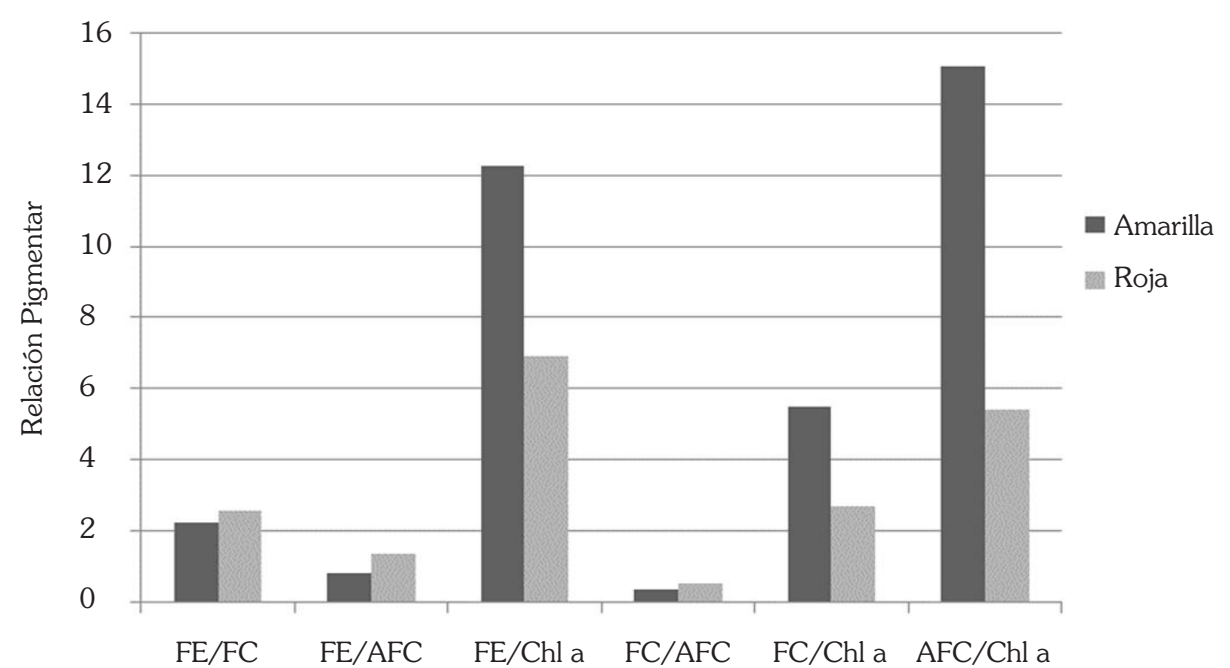

Fig. 4. Relación pigmentar de variantes rojas y amarillas de A. plicata, colectadas del Sector de Seno Skyring, XII Región. (AFC: aloficocianina, FC: ficocianina, FE: ficoeritrina, $\mathrm{Cl} a$ : clorofila a).

y roja, por el contrario, en las estaciones con mayor fertilidad (primavera), la mayoría de los talos suelen poseer una coloración roja. Observandose una relación asociada con la marcada estacionalidad en zonas de altas latitudes, principalmente influida por fotoperiodo e intensidad de radiación solar que determinan variaciones en macroalgas (Ojeda et al. 2014). Esta caracterización de las variantes de A. plicata, podría profundizarse a futuro en base a estudios morfológicos a nivel celular y moleculares para definir si las variantes de color, representan expresiones genéticas de origen nuclear o cloroplastidial como definido para otras especies de la División Rhodophyta como referido por Guimarães (2000); Ursi y Plastino (2001).

Neveux et al. (2006), señala que la ficoeritrina es la responsable de otorgarle el color rojo a las algas, este pigmento accesorio juega un importante rol en la captación de la luz en macroalgas rojas. Además de ser una de las reservas más importantes de nitrógeno en las algas (Martinez \& Rico, 2006). Los resultados del presente estudio coinciden con los obtenidos en el trabajo realizado por Fernandes et al. (2012), en Hypnea musciformes (Wulfen) J. V. Lamouroux, 1813, cuya concentración de ficoeritrina es predominante tanto en condiciones de cultivo como en condiciones naturales. En el estudio realizado por Costa et al. (2001), los ejemplares de Gracilaria birdae de coloración roja, verde- marrón y verde, colectados en un mismo ambiente y posteriormente sometidos a un cultivo, se obtuvo que los ejemplares de mayor crecimiento fueron los de coloración roja. Resultado de deficiencias metabólicas, característica en mutantes de coloración verde, lo que disminuye su eficiencia fotosintética, traducido en un menor crecimiento. Para Niwa et al. (2002), los valores de ficoeritrina y las relaciones $\mathrm{FE} / \mathrm{Chl}$ a y $\mathrm{FE} / \mathrm{FC}$ son mayores para el tipo silvestre rojo en comparación al tipo mutante verde de la especie Porphyra yezoensis Ueda (1932), por lo tanto, infieren principalmente a una variación cuantitativa de ficobilinas. Estos resultados coinciden con los obtenidos en A. plicata, con valores mayores en las relaciones FE/FC y FE/AFC para las variantes rojas. De acuerdo a lo señalado por Yokoya et al. (2007), las concentraciones de ficobiliproteínas, y principalmente la ficoeritrina, se encuentra estrechamente relacionado a factores ambientales como los niveles de irradiación y la eficiencia fotosintética, lo que probablemente pueda estar ocurriendo con la variante roja de A. plicata, como una variante mucho más eficiente en absorber energía luminosa, a diferencia de la variante amarilla.

Los resultados permiten concluir que la variante roja de A. plicata presentó las mayores concentraciones de pigmentos fotosintéticos en base a ficobiliproteínas y clorofila $\alpha$. De acuerdo 
al mayor valor de ficoeritrina obtenido para la variante roja, se puede indicar que es la variante mas eficiente fotosintéticamente.. También es importante tener en cuenta las condiciones de luz a las que se enfrenta $A$. plicata, como la marcada estacionalidad que se produce en estos ambientes de altas latitudes, y que genera la necesidad de adaptaciones pigmentares de las especies para la captación eficiente de energía luminosa. Por último, cabe mencionar que estas coloraciones (amarilla y roja) pueden ser efecto de variaciones genéticas intraespecíficas en ejemplares de $A$. plicata. (Yokoya et al. 2007; Martins \& Yokoya, 2010).

El presente estudio sobre la composición pigmentar de las variantes amarillas y rojas de A. plicata, contribuye al conocimiento fisiológico en variantes de A. plicata y proporciona nuevos antecedentes para futuros estudios genéticos $y$ biotecnológicos de esta especie de valor comercial presente en la región de Magallanes,.

\section{AGRADECIMIENTOS}

Los autores agradecen el respaldo de Fondos Basales - CONICYT, PO5-002 (ICM-MIDEPLAN), Proyecto Fondef DO8I1163, y Propuesta Atracción MEC 80100021 de CONICYT a la Dra. Nair Yokoya - Ibt - SP, Brasil. El presente estudio cuenta con el apoyo y financiamiento del proyecto FONDECYT 1140940 "Macroalgal adaptive radiation: potential links to ecological niche diversity in the ecoregion of Magallanes and Chilean Antarctic". Johanna Marambio, Juan Pablo Rodríguez y Fabio Méndez agradecen a la beca entregada por el Instituto de Ecología y Biodiversidad (IEB), código PFB-23-2008 y código ICM P05-002.

\section{LITERATURA CITADA}

Avila, M., Villanueva, F., Yokoya, N., \& Mansilla, A. (2012). Manual de cultivo de Ahnfeltia plicata (Hudson) Fries en base a fitohormonas (reguladores de crecimiento). Serie Programa educativo para el desarrollo de la acuicultura de especies nativas: Ahnfeltia plicata. Universidad Arturo PratUniversidad de Magallanes.
Ben-Amotz, A., Katz, A., \& Avron, M. (1982). Accumulation of beta-carotene in halotolerant algae: purification and caracterization of beta-carotene rich globules from Dunaliella bardawil (Chlorophyceae). Journal of applied Phycology, 25, 175178.

Borowitzka, L. J., Borowitzka, M .A., \& Kessley, D. (1990). Effects of salinity increase on carotenoid accumulation in the green algae Dunaliella salina. Journal of applied Phycology, 2, 111-119.

Costa, V. L., \& Plastino, E. M. (2001). Histórico de vida de espécimes selvagens $e$ variantes cromáticas de Gracilaria birdiae (Gracilariales, Rhodophyta). Revista Brasileira de Botanica, 24, 491-500

Costa, V. L., \& Plastino, E. M. (2011). Color inheritance and pigment characterization of red (wild-type), greenish-brown, and green strains of Gracilaria birdiae (Gracilariales, Rhodophyta). Journal of Applied Phycology, 23, 599-605.

Fernandes, D. R., Caetano, V. S., Tenório, M., Reinert, F., \& Yoneshigue-Valentin, Y. (2012). Characterization of the photosynthetic conditions and pigment profiles of the colour strains of Hypnea musciformis from field-collected and in vitro cultured samples. Revista Brasileira de Farmacognosia, 22(4), 753-759.

Gantt, E. (1990). Pigmentation and photoacclimation. En K. M. Cole \& R. G. Sheath (Eds.), Biology of the red algae (pp 203-219). Cambridge University Press, Cambridge.

Gerung, G. S., \& Ohno, M. (1997). Growth Rates of Eucheuma denticulatum (Burman) Collins et Harvey and Kappaphycus striatum (Schmitz) Doty under Diferent Conditions in Warm Waters of Southern Japan. Journal Applied of Phycology, 9, 413-415.

Gómez, E. (2013). Evaluación nutricional y propiedades biológicas de algas marinas comestibles. Estudios "in vitro" $e$ "in vivo". Tesis de Doctorado. Madrid, España: Departamento de Nutrición y Bromatología II (Bromatología), Facultad de Farmacia, Universidad Complutense de 
Madrid.

Guimarães, M. (2000). Aspectos fisiológicos de Gracilaria domingensis (Gracilariales, Rhodohphyta): subsídios para a compreensão da manutenção do polimorfismo pigmentar. Tesis Doctorado. Sao Paulo, Brasil: Departamento de Botánica, Instituto de Biociencias de la Universidad de Sao Paulo.

Guimarães, M., Plastino, E. M., \& Destombe, C. (2003). Green mutant frequency in natural population of Gracilaria domingensis (Gracilariales, Rhodophyta) from Brazil. European Journal of Phycology, 38(2), 165-169.

Jefrey, S. W., \& Hunphrey, G. F. (1975). New spectrophotometric equation for determining chlorophylls a, b c1 and c2 in higher plants, algae and natural phytoplanckton. Biochemie und Physiologie der Pflanzen, 167, 191-4.

Kain (Jones), J. M., \& Destombe, C. (1995). A review of the life history, reproduction and phenology of Gracilaria. Journal of Applied Phycology, 7, 269-281.

Kirk, J. T. (1994). Light and photosynthesis in aquatic ecosystems. Cambridge university press.

Kursar, T. A., Van der meer, J. P., \& Aberte, R. S. (1983a). Light-harvesting system of the red alga Gracilaria tikvahiae. I. Biochemical analyses of pigment mutations. Plant Physiology, 73, 353-360.

Kursar, T. A., Van der Meer, J., \& Alberte, R. S. (1983b). Light-harvesting system of the red alga Gracilaria tikvahiae II. Phycobilisome characteristics of pigment mutants. Plant Physiology, 73, 361-369.

Maggs, C. A., \& Pueschel, C. M. (1989). Morphology and development of Ahnfeltia plicata (Rhodophyta): proposal of Ahnfeltiales ord. nov. 1. Journal of applied Phycology, 25(2), 333-351.

Mansilla, A., \& Navarro, N. (2003). Contribución al estudio de la flora ficológica de las Islas Diego Ramírez (Chile). Memorias de Curso Internacional de Postgrado y Especialización de macroalgas en ambientes subantárticos. Ediciones Universidad de Magallanes.
Mansilla, A., Ávila, M., \& Yokoya, N. S. (2012). Current knowledge on biotechnological interesting seaweeds from the Magellan Region, Chile. Revista Brasileira de Farmacognosia, 22(4), 760-767.

Mansilla, A., Rodriguez, J. P., Souza, J. M., Rosenfeld, S., Ojeda, J., \& Yokoya, N. S. (2013). Growth responses to temperature, salinity and nutrient variations, and biomass variation and phenology of Ahnfeltia plicata (Rhodophyta, Ahnfeltiales): a commercially interesting agarophyte from the Magellanic Region, Chile. Journal of applied phycology, 26(2), 1133-1139.

Martínez, B., \& Rico, J. M. (2002). Seasonal variation of $\mathrm{P}$ content and major $\mathrm{N}$ pools in Palmaria palmata (Rhodophyta). Journal of Phycology, 38, 1082-1089.

Martins, A. P., \& Yokoya, N. S. (2010). Intraspecific variations in colour morphs of Hypnea musciformis (Rhodophyta) in relation to nitrogen availability. Hoehnea, 37(3), 601615.

Matsuhiro, B., Barahona, T., Encinas, M. V., Mansilla, A., \& Ortiz, J. A. (2014). Sulfation of agarose from subantarctic Ahnfeltia plicata (Ahnfeltiales, Rhodophyta): studies of its antioxidant and anticoagulant properties in vitro and its copolymerization with acrylamide. Journal of Applied Phycology, 26(5), 2011-2019.

Motten, A. F. (1995). Diversity of photosynthetic pigments. En C. A. Goldman (Ed.), Tested Studies for Laboratory Teaching. Vol. 16. (pp. 81-98). Proc. 16th Workshop/ Conference of the Association for Biology Laboratory Education.

Neveux, J., \& Lantoine, F. (1993). Spectrofluorometric assay of chlorophylls and phaeopigments using the least squares approximation technique. DeepSea Research: Oceanographic Research Papers, 40, 1747-1765.

Neveux, J., Tenório, M. M. B., Dupouy, C., \& Villareal, T. A. (2006). Spectral diversity of phycoerythrins and diazotroph abundance in tropical waters. Limnol Oceanogr, 51, 1689-1698.

Niwa, K., Mizuta, A., \& Aruga, Y. (2002). Genetic 
characterization of a spontaneous Greentype pigmentation mutant of Porphyra yezoensis and the significance of using heterozygous conchocelis in nori farming. Fisheries Science, 68, 729-735.

Ojeda, J., Rosenfeld, S., Marambio, J., Rozzi, R., \& Mansilla, A. (2014). Patrones estacionales y espaciales de la diversidad de moluscos intermareales de bahía Róbalo, canal Beagle, Reserva de la Biosfera Cabo de Hornos, Chile. Revista de biología marina y oceanografía, 49(3), 493-509.

Plastino, E. M., \& Guimarães, M. (2001). Diversidad intraespecífica. En K. V. Alveal \& T. J. Antezana (Eds.), Sustentabilidad de la Biodiversidad (pp. 19-27). Concepción, Universidad de Concepción.

Plastino, E., \& Mansilla, A. (2004). Luzy fotosíntesis. En C. Werlinger (Ed.), "Biología Marina y Oceanografía: Conceptos y Procesos" (pp. 229-252). Consejo Nacional del Libro y la Lectura - Universidad de Concepción. Trama Impresores S.A.

Ramus, J., \& van der Meer, J. P. (1983). A physiological test of the theory of complementary chromatic adaptation I. Color mutants of a red seaweed. Journal of applied phycology, 19, 86-91.

Ursi, S., \& Plastino, E. M. (2001). Crescimento in vitro de linhagens de coloração vermelha $e$ verde clara de Gracilaria sp. (Gracilariales, Rhodophyta) em dois meios de cultura: análise de diferentes estádios reprodutivos. Revista Brasileira de Botânica, 24(4), 587594.

Werlinger, C. (2004). Biología Marina y Oceanografía: Conceptos y Procesos (Marine Biology and Oceanography): Concepts and processes. Fondo Nacional del libro y a la lectura. Editorial Trama.

Wiencke, C., \& Clayton, M. N. (2002). Antarctic Seaweeds. In: Wägelee, J. W. (Ed.), Synopses of the Antarctic Benthos, 9 (159 pp), 40 pls.

Yokoya, N. S., Plastino, E. M., Artel, R. (2003). Physiological responses and pigment characterization of two colour strains of the carrageenophyte Hypnea musciformis (Rhodophyta). En A. R. O. Chapman, R. J. Anderson, V. J. Vreeland, I. R. Davison(Eds.), Proceedings of the 17th International Seaweed Symposium (pp, 425-433) New York: Oxford University Press,

Yokoya, N. S., Necchi Júnior, O., Martins, A. P., Gonzalez, S. F., \& Plastino, E. M. (2007). Growth responses and photosynthetic characteristics of wild and phycoerythrindeficient strains of Hypnea musciformis (Rhodophyta). Journal of applied phycology, 19, 197-205.

Zhang X. C., \& Van Der Meer, J. P. (1987). A study on heterosis in diploid gametophytes of the marine red alga Gracilaria tikvahiae. Botanica Marina, 30, 309-314. 
